\title{
THE APRIL MEETING OF THE CHICAGO SECTION.
}

THE seventh regular meeting of the Chicago Section of the American Mathematical Society was held at Northwestern University, Evanston, Ill., on Saturday, April 14, 1900. The morning session was called to order at ten o'clock, by Professor E. H. Moore, Vice-President of the Society.

The following members were present:

Professor E. W. Davis, Professor Thomas F. Holgate, Mr. H. G. Keppel, Dr. Kurt Laves, Professor H. Maschke, Professor E. H. Moore, Professor James B. Shaw, Professor E. B. Skinner, Dr. H. F. Stecker, Professor H. S. White, Professor J. W. A. Young.

Professor James B. Shaw was elected chairman for the meeting, which extended through a forenoon and an afternoon session.

Papers were presented as follows :

(1) Professor E. H. Moore : "Evaluation of an important determinant of special form."

(2) Professor H. MAschke : "A new method of determining the differential parameters and invariants of quadratic differential quantics."

(3) Mr. Robert E. Moritz: "On the transcendency of $\pi . "$

(4) Dr. H. F. BlichFeld : "Note on the functions of a given class which in a given interval differ the least possible from zero."

(5) Dr. KuRT Laves: "Maupertuis's principle of least action for forces involving the coördinates and their first and higher differential quotients."

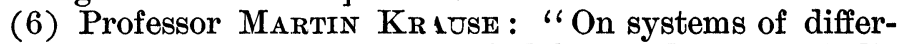
ential equations which are satisfied by quadruply periodic functions."

(7) Professor James B. Shaw : "Multiple algebra."

(8) Professor E. W. Davis : "A graphic method for the solution of systems of linear equations."

(9) Professor L. E. Dickson: "Determination of an abstract group of order $2^{7} .3^{6} .5 .7$ holoedrically isomorphic with a certain orthogonal group and with a certain hyperabelian group."

(10) Professor E. H. Moone : "On the generational determination of abstract groups."

(11) Professor H. B. Newson : "On the group of 216 collineations of the plane." 
(12) Mr. James Archy Smith : "Definition and periodicity of the abstract group holoedrically isomorphic with a certain group of algebraic transformations."

(13) Professor E. W. DAvis : "On the use of color in the representation of crystal groups."

Mr. Moritz's paper was presented to the Society and read by Professor Davis ; Dr. Blichfeldt's and Mr. Smith's papers were presented through Professor Moore, and the former was also read by Professor Moore; Professor Krause's paper was presented to the Society and read by Professor Maschke. Professor Newson's paper was read by title. Abstracts of the papers are given below.

Professor Moore's first paper, which has been offered for publication to the Annals of Mathematics, contains, after certain generalities on determinantal notation, four proofs of the theorem that the determinant

$$
\left|a_{g i, h j}\right| \quad\left(\begin{array}{c}
g, h=1, \cdots, m \\
i, j=1, \cdots, n
\end{array}\right)
$$

of order $m n$, where throughout

$$
a_{g i, h j}=b_{g h}^{(i)} c_{i j}^{(h)},
$$

is the continued product of the $n$ determinants $B^{(i)}$ of order $m$ and the $m$ determinants $C^{(h)}$ of order $n$

$$
B^{(i)}=\left|b_{g h}^{(i)}\right|, \quad C^{(h)}=\left|c_{i j}^{(h)}\right| \cdot
$$

This theorem, stated in a different notation, was first discovered by Professor W. H. Metzler.

Professor Maschke's paper presents a symbolic method for the treatment of differential parameters and differential invariants. If $F$ and $\Phi$ are any two invariants ( $i$. e., differential parameters or differential invariants) of the binary quadratic differential quantic

then the expression

$$
A=\sum_{r, s=1}^{2} a_{r s} d x_{r} d x_{s},
$$

$$
\sqrt{\overline{a_{11} a_{22}-a_{12}^{2}}}\left|\begin{array}{ll}
\frac{\partial F}{\partial x_{1}} & \frac{\partial F}{\partial x_{2}} \\
\frac{\partial \Phi}{\partial x_{1}} & \frac{\partial \Phi}{\partial x_{2}}
\end{array}\right|=\{F, \Phi\}
$$


is again an invariant of $A$. Let $f, \varphi, \cdots, u, v, \cdots$ be arbitrary functions of $x_{1}, x_{2}$; as such they are invariants of $A$, and consequently the expressions

$$
\{f, u\}, \quad\{f,\{\varphi, u\}\}, \quad\{\{f, u\},\{\varphi, v\}\}, \quad \text { etc. },
$$

will be invariants of $A$. If now we agree to set

we obtain

$$
\frac{\partial f}{\partial x_{r}} \cdot \frac{\partial f}{\partial x_{s}}=a_{r s},
$$

$$
\left(\frac{\partial f}{\partial x_{1}} d x_{1}+\frac{\partial f}{\partial x_{2}} d x_{2}\right)^{2}=\Sigma a_{r s} d x_{r} d x_{s}=A,
$$

and we call $f$ a symbol of $A$. It follows then, as in algebra, that every product of expressions (1) containing each symbol $f, \varphi, \cdots$ precisely twice (with certain restrictions concerning the higher derivatives) will be an actual invariant of $A$. Thus the following invariants are obtained :

$\{f, u\}^{2}=\Delta_{1} u,\{f, u\}\{f, v\}=\nabla(u, v),\{f,\{f, u\}\}=\Delta_{2} u$, $\{f, \varphi\}\{\{f, u\},\{\varphi, u\}\}=2 \triangle_{22} u,\{\varphi, \psi\}\{\{f, \varphi\},\{f, \phi\}\}=2 K$.

The extension of the method from the case of binary differential quantics to that of $n$ variables, from quadratic quantics to those of higher orders, from invariants to covariants, and also from invariants and covariants of one quantic to those of two or more quantics, is obvious. The full paper will appear in the Transactions.

To prove that $\pi$ is transcendental it is sufficient to show that $e$ cannot satisfy an equation of the form

$$
C_{0}+e^{a_{1}}+e^{a_{2}}+\cdots+e^{a_{3}}=0,
$$

where $C_{0}$ is an integer and the $\alpha$ 's are associated algebraic numbers. Hurwitz has shown that, for every rational function $f(x)=0$, another function $F(x)=0$ exists, such that

$$
F(x)-e^{x} F(0)=-x e^{(1-\theta) x} f(\theta x) \quad(0<\theta<1) .
$$

Mr. Moritz points out that by putting

$$
f(x)=\frac{x^{p-1}}{(p-1) !} \cdot a_{0}^{n p-1} \cdot \prod_{i=1}^{n}\left(\alpha_{i}-x\right)^{p}
$$

the assumption [A] leads to the equation

$$
C_{0} F(0)+\sum_{i=1}^{n} F\left(\alpha_{i}\right)=\sum_{i=1}^{n}\left[-\alpha_{i} e^{(1-\theta) \alpha_{i}} f\left(\theta \alpha_{i}\right)\right],
$$


in which $C_{0} F(0)$ is not divisible by the integer $p, \Sigma F\left(\alpha_{i}\right)$ is divisible by $p$, and the right member of the equation approaches zero as a limit with increasing values of $p$. This is absurd; hence $[A]$ does not exist.

A real function of a real variable and certain real parameters being given, Tschebycheff has treated the problem of determining the parameters so that in a given interval the maximum absolute value of the function shall be as small as possible. In Dr. Blichfeldt's paper, which has been offered to the Transactions for publication, the necessary conditions on the parameters which Tschebycheff exhibited are found, when supplemented by certain others, to be also sufficient conditions.

Dr. Laves shows that the theorem of Maupertuis can be proved to exist for certain forces of the general nature indicated in the title of his paper which possess an effective potential $W$. The potential $W$ is an arbitrary function of the coördinates and velocities of the bodies involved, not containing the time $t$ explicitly. The differential equations of motion for effective potential forces are, in Lagrange's second form,

$$
\frac{d}{d t}\left(\frac{\partial(T+W)}{\partial q_{i}^{\prime}}\right)-\frac{\partial}{\partial q_{i}}(T+W)=0 \quad(i=1, \cdots, k),
$$

where $T$ is the kinetic energy of the system. Starting from Jacobi's partial differential equation of the first order

$$
\frac{\partial v}{\partial t}-K\left(q_{1}, \cdots, q_{k}, \frac{\partial v}{\partial q_{1}}, \cdots, \frac{\partial v}{\partial q_{k}}\right)=0,
$$

we assume the complete integral

$$
v=-h t+\bar{v}\left(q_{1}, \cdots, q_{k}, a_{1}, \cdots, a_{k-1}\right)+a_{k}
$$

and define the action of the system during the interval of time $t_{1}-t_{0}$ by

$$
A=\int d v=\int_{t_{0}}^{t_{1}} \Sigma \frac{\partial \bar{v}}{\partial q_{1}} q_{1}^{\prime} d t=\int_{t_{0}}^{t_{1}} \Sigma p_{i} q_{i}^{\prime} d t
$$

where

$$
p_{i}=\frac{\partial(T+W)}{\partial q_{1}^{\prime}} \text {. }
$$

Calling $T+W=\varphi$, we make the special assumption that $\varphi=\varphi_{0}+\varphi_{1}+\varphi_{2}$, where $\varphi_{0}, \varphi_{1}, \varphi_{2}$ are homogeneous functions 
of $q_{i}^{\prime}$ of the degree indicated by the sulsscript in each case. The integral of the conservation of vis viva is

$$
K \equiv \Sigma q_{i}^{\prime} \frac{\partial(T+W)}{\partial q_{i}^{\prime}}-(T+W) \equiv \varphi_{2}-\varphi_{0}=h .
$$

The expression for $A$ becomes

$$
A=\int_{t_{0}}^{t_{1}}\left(2 \varphi_{2}+\varphi_{1}\right) d t
$$

We may eliminate $d t$ by means of the equation $\varphi_{2}=\varphi_{0}+h$ by considering

$$
\varphi_{2}=\sum a_{i j} \frac{d q_{i} d q_{j}}{d t^{2}} \equiv \frac{d \sigma^{2}}{d t^{2}}
$$

where we have put $d \sigma^{2} \equiv \sum a_{i j} d q_{i} d q_{j}$. Thus we obtain

$d t=\frac{d \sigma}{\sqrt{\varphi_{0}+h}} \cdot \quad$ Putting $2 \varphi_{2}=2\left(\varphi_{0}+h\right)$ and $\varphi_{1} \equiv \Sigma \frac{b_{i} d q_{i}}{d t} \equiv \frac{d \tau}{d t}$ we obtain $A=\int_{\sigma_{0}}^{\sigma_{1}} 2 \sqrt{\varphi_{0}+h} d \sigma+\int_{\tau_{0}}^{\tau_{1}} d t$. It is easy to

show that the condition $\delta A=0$ is fulfilled for the actual path described by the system. In fact, putting $q_{1}=f_{1}(\lambda), \cdots$, $q_{k}=f_{k}(\lambda)$, we can express $A$ as a function of the parameter $\lambda$ as follows :

$$
A=\int_{\lambda_{0}}^{\lambda_{1}}\left\{2 \sqrt{\varphi_{0}+h} \sqrt{\sum a_{i j} \cdot \dot{q}_{i} \cdot \dot{q}_{j}}+\sum b_{i} \dot{q}_{i}\right\} d \lambda
$$

where we have put $q_{i}=\frac{d q_{i}}{d \lambda}$.

Performing the variation of $A$, and remembering that the coordinates are given for $\lambda=\lambda_{0}$ and $\lambda=\lambda_{1}$, we obtain after an integration by parts and re-introduction of the variable $t$

$$
\delta A=\int_{\tau_{0}}^{\tau_{1}} \Sigma L_{i} \delta q_{i} d t
$$

The coefficients $L_{i}$ present themselves in the form

$$
L_{i}=\frac{d}{d t}\left(\frac{\partial(T+W)}{\partial q_{i}^{\prime}}\right)-\frac{\partial}{\partial q_{i}}(T+W) .
$$

They vanish in accordance with our hypothesis, and it is 
thus proved, that the condition $\delta A=0$ is fulfilled for the case under consideration.

In the theory of elliptic functions certain linear differential equations prove to be of importance, the coefficients of which are doubly periodic, while the integrals can also be represented as doubly periodic functions. In particular, two equations have been investigated, Lamé's and Picard's differential equations of the second order. In previous papers Professor Krause established a system of differential equations as an analogue of Lamé's equation. In his present paper he exhibits as the simplest analogue of Picard's equation a system of two linear differential equations, the coefficients and also the integrals of which are quadruply periodic functions.

Professor Shaw's paper is a complete generalization of quaternions to an algebra of $n$ units. This is rendered possible by considering what has heretofore been called a product of two multiple numbers as simply a function of two such numbers, or of two sets of $n$ variables. By the introduction of the symbol $D$ defined by

where

$$
\begin{gathered}
\overline{D \bar{\alpha} \beta}=a_{1} b_{1}+a_{2} b_{2}+\cdots+a_{n} b_{n}, \\
\alpha=\sum_{1}^{n} a_{i} e_{i}, \quad \beta=\sum_{1}^{n} b_{i} e_{i},
\end{gathered}
$$

the $e^{\prime}$ s being defining units; and by the introduction of a set of $A$ 's so defined that

$$
A . \alpha_{1} \alpha_{2} \cdots \alpha_{r} A . \beta_{1} \cdots \beta_{r+1}=\left|\begin{array}{ccc}
\beta_{1} \cdots & \beta_{r+1} \\
D \bar{\alpha}_{1} \beta_{1} & \cdots & D \overline{\alpha_{1}} \beta_{r+1} \\
\cdots \ldots \ldots \ldots \ldots \ldots \ldots \ldots \\
\cdots \cdots \cdots \cdots \cdots \cdots \cdots . . \\
D \overline{\alpha_{r}} \beta_{1} & \cdots & \bar{D} \bar{\alpha}_{r} \beta_{r+1}
\end{array}\right|,
$$

every quaternion formula finds its extension. The paper includes the theory of matrices of order $n$ as the linear vector operators of such algebras. It is shown that every such matrix may be written in the form $\varphi=\sum_{1,1}^{n, n} a_{r s} v_{01}{ }^{8} v_{10}{ }^{r}$, where $v_{01}$ and $v_{10}$ are matrices of order $n, v_{01}{ }^{n}=1, v_{10}{ }^{n}=1$, $v_{01}{ }^{8} v_{10}{ }^{r}=i^{-r s} v_{10}{ }^{r} v_{10}{ }^{8}, i$ being a primitive $n$th root of unity. Just as in quaternions $(1+\sqrt{-1} k) i=i+\sqrt{-1} j$ is a nullity, so in matrices in general $\theta_{r-1}=\left(1-v_{10}\right)\left(1-i v_{10}\right)$ $\left(1-i^{2} v_{10}\right) \cdots\left(1-i^{r-2} v_{10}\right)\left(1-i^{r} v_{10}\right) \cdots\left(1-i^{n-1} v_{10}\right) v_{01}$ is a 
nullity, $i . e_{.}, \theta_{r-1}^{2}=0$. Any expression $\theta=\theta_{a}+\theta_{b}+\cdots$ $+\theta_{f}$ is also a nullity, $i$. $e_{.}, \theta^{p}=0, p$ being an integer less than $n$. A complete list of expressions for the moduli, etc., of matrices and of combined matrices is given.

Professor Davis presented the following graphic method for the solution of a system of linear equations. Let (1), (2), (3), (4) stand for four equations in $x, y, z, w$; let (12) stand for the result of eliminating $z$, and $(12)^{\prime}$ for the result of eliminating $w$ from (1) and (2); while (123) is the result of eliminating both $z$ and $w$ from (1), (2), and (3). Further, let (1) $a b$ be the graph in the $x y$-plane of the result of putting $z=a$ and $w=b$ in (1), while (12) is the graph of the result of putting $w=a$ in (12). To solve the equations draw $(1)_{00}$ and $(2)_{00},(1)_{10}$ and (2) ${ }_{10}$. Each pair meets upon (12), which draw. Similarly determine and draw $(13)_{0}$ together with (12) ${ }_{1}$ and (13) $)_{1}$. Each pair meets upon the graph of (123). Draw this graph and likewise determine and draw the graph of (124). These two graphs meet in $P$ whose coördinates are the proper values of $x$ and $y$ to satisfy the original equations. Finally $w$ equals the distance from $(12)_{0}$ to $P$ divided by the distance from $(12)_{0}$ to $(12)_{1}$, while the value of $z$ is obtained by accenting the parentheses in the value of $w$. The method can be extended to any number of equations in as many unknowns.

The groups referred to in the title of Professor Dickson's paper are included in the author's list of simple groups published in the Bulletin for July, 1899. The paper will appear in the Transactions.

Besides fundamental generalities (cf. Bulletrn, December, 1899, p. 99), the second paper of Professor Moore, which is to appear in the Transactions, develops certain examples of which the most important is the following: The group $F_{2, R, 1}$ of binary fractional matrices $\left(\frac{a, b}{c, d}\right)$, $(a d-b c=1)$, of determinant 1 in any field or realm $R$ of rationality, has the generators

$$
T=\left(\frac{0,-1}{1,-0}\right), \quad S_{s}=\left(\frac{1, s}{0,1}\right)
$$

$s$ being any mark of the realm $R$; and the corresponding abstract group is given in the abstract generator symbols $T, S_{s}$, by the system of generational relations :*

* Another generational determination of this group was given at this meeting in the paper of Mr. J. A. Smith. 


$$
T^{2}=I, \quad \text { （2） } S_{0}=I, \quad \text { （3） } \quad S_{s_{1}} S_{s_{2}}=S_{s_{1}+s_{2}}
$$

(4) $S_{s_{1}} T S_{s_{2}} T S_{s_{3}} T S_{s_{4}} T S_{s_{5}} T=I$,

where $I$ is the identity element, and in (3) $s_{1}, s_{2}$ are any two marks of the realm $R$, and in (4) $s_{1}, s_{2}$ are any two nonreciprocal marks of the realm $R$, and

$$
s_{3}=\frac{s_{1}-1}{s_{1} s_{2}-1}, \quad s_{4}=-\left(s_{1} s_{2}-1\right), \quad s_{5}=\frac{s_{2}-1}{s_{1} s_{2}-1} .
$$

It should be noted that the marks $s_{1}, \cdots, s_{5}$ are the roots of a quintic equation whose coefficients are rational functions with integral coefficients of two arbitrary parameters and which in the realm of its coefficients is solvable by radicals.

Professor Newson investigates the geometrical properties of the group of 216 collineations in the plane and its subgroups with respect to a pencil of cubic curves remaining invariant under the group. In particular he discusses the effect of the transformations of the different subgroups on the harmonic and equianharmonic cubics and the four inflexional triangles contained in the pencil and their relation to the invariant triangles belonging to the transformations. The structure of the group is analogous to that of the tetrahedron group, the four equianharmonic cubics, the four degenerate cubics, and the six harmonic cubics of the pencil being analogous to the four vertices, four faces, and six edges of the tetrahedron.

Mr. Smith's paper exhibits in the first place certain novel generators of linear substitution groups which may be either homogeneous and integral in two letters or fractional in one. The determinant is taken as unity and the parameters are taken either $1^{\circ}$ integers modulo $q, 2^{\circ}$ marks of a Galois field of order $q^{n}$ together with integers modulo $q$, or $3^{\circ}$ continuous variables either real or complex. For each of these three fields two types of generating substitutions $S_{\alpha}$ and $V_{\alpha}$ are defined, and relations are found which suffice for complete definition of the isomorphic abstract groups. These generators are so defined in each of the fields above mentioned that the formal relations are the same for all, the fractional linear group requiring three relations, the integral only two. In the second part of the paper normal representations of all elements of the groups are given in terms of generating elements, and their periodicity is determined by means of these normal forms. 
In Professor Davis's second paper it is pointed out that the 48 operations which bring the cube into coincidence with itself are operations which bring any one of the eight triangles into which a face is divided by its lines of symmetry into coincidence with the 48 like triangles of the surface of the cube. Any of the subgroups of operations brings this same triangle into coincidence with merely a part of the 48 triangles. A cube with these triangles all of one color and the rest of the cube another color is an object which, taking account of color, comes into coincidence with itself only under the operations of the subgroup. Similar methods can be used for the representation of all regular groups and their subgroups.

Thomas F. Holgate, Secretary of the Section.

\section{ON THE HISTORY OF THE EXTENSIONS OF THE CALCULUS.}

BY J. G. HAGEN, S. J.

THE results attained by means of the infinitesimal calculus naturally evoked similar attempts in other directions. We may distinguish between two kinds of new theories, differing from each other mainly in their origin. The one is the natural outcome of an ever recurring need of solving practical problems or of giving existing theories a broader basis; the other owes its origin to purely abstract speculation. The former is not the property of any one mathematician; its nomenclature is not arbitrarily chosen, and its general introduction is only a question of time. This is clear from the history of all those branches of mathematics that have come into general use.

The case is somewhat different with the purely speculative theories. But though these may never succeed in demonstrating their practical utility, yet they are of value to mathematical science since they throw new light on existing methods and on their usefulness. For our purpose, however, a summary outline of these theories will suffice.

The present abstract is confined to those theories that are in close relation to the infinitesimal calculus and the theory of functions, and excludes, for instance, all geometrical methods and what are called "principles" or methods of demonstration. 